\title{
Mediterranean Diet and KNEE Osteoarthritis Outcomes: a Longitudinal Cohort Study
}

\author{
Nicola Veronese, $\mathbf{M D}^{1,2}$, Ai Koyanagi, MD $^{3,4}$, Brendon Stubbs, $\mathrm{PhD}^{5,6,7}$, Cyrus Cooper, \\ MD ${ }^{8,9,10}$, Giuseppe Guglielmi, MD ${ }^{11,12}$, Renè Rizzoli, MD ${ }^{13}$, Leonardo Punzi, MD ${ }^{14}$, \\ Domenico Rogoli, MD , Maria Gabriella Caruso, MD², Ornella Rotolo, RD², Maria \\ Notarnicola, ScD ${ }^{2}$, Nasser Al-Daghri, MD ${ }^{15}$, Lee Smith, PhD ${ }^{16}$, Jean-Yves Reginster, MD, \\ $\mathrm{PhD}^{15,17}$, and Stefania Maggi, MD ${ }^{1}$
}

${ }^{1}$ National Research Council, Neuroscience Institute, Aging Branch, Padova, Italy ${ }^{2}$ National Institute of Gastroenterology-Research Hospital, Castellana Grotte, Bari, Italy ${ }^{3}$ Research and Development Unit, Parc Sanitari Sant Joan de Déu, Universitat de Barcelona, Fundació Sant Joan de Déu, Barcelona, Spain ${ }^{4}$ Instituto de Salud Carlos III, Centro de Investigación Biomédica en Red de Salud Mental, CIBERSAM, Madrid, Spain ${ }^{5}$ Physiotherapy Department, South London and Maudsley NHS Foundation Trust, Denmark Hill, London SE5 8AZ, UK ${ }^{6}$ Health Service and Population Research Department, Institute of Psychiatry, Psychology and Neuroscience King's College London, De Crespigny Park, London Box SE5 8AF, UK ${ }^{7}$ Faculty of Health, Social Care and Education, Anglia Ruskin University, Bishop Hall Lane, Chelmsford CM1 1SQ, UK ${ }^{8}$ Oxford NIHR Musculoskeletal Biomedical Research Unit, Nuffield Department of Orthopaedics, Rheumatology and Musculoskeletal Sciences, Nuffield Orthopaedic Centre, University of Oxford, Windmill Road, Oxford, OX3 7LD, UK ${ }^{9}$ MRC Lifecourse Epidemiology Unit, Southampton General Hospital, University of Southampton, Southampton, SO16 6YD, UK ${ }^{10}$ National Institute for Health Research Nutrition Biomedical Research Centre, University of Southampton and University Hospital Southampton NHS Foundation Trust, Southampton General Hospital, Southampton, SO16 6YD, UK ${ }^{11}$ Department of Radiology, University of Foggia, Foggia, Italy ${ }^{12}$ Department of Radiology, Scientific Institute "Casa Sollievo della Sofferenza" Hospital, San Giovanni Rotondo, Foggia, Italy ${ }^{13}$ Division of Bone Diseases, Department of Internal Medicine Specialties, Geneva University Hospitals and Faculty of Medicine, Geneva, Switzerland ${ }^{14}$ Rheumatology Unit, Department of Medicine (DIMED), University of Padova, Padova, Italy ${ }^{15}$ Prince Mutaib Chair for Biomarkers of Osteoporosis, Biochemistry Department, College of Science, King Saud University, Riyadh, Saudi Arabia ${ }^{16}$ The Cambridge Centre for Sport and Exercise Sciences, Anglia Ruskin

\footnotetext{
Corresponding Author: Nicola Veronese, MD, National Research Council, Neuroscience Institute, Aging Branch, Via Giustiniani, 2 35128 Padova, Italy, Phone: +390498211746; Fax: +390498211218, ilmannato@gmail.com.

Declarations of interest: Dr. Rizzoli reports personal fees from Radius Health, Sandoz, Labatec-Effryx, Mylan; Dr. Reginster reports grants and personal fees from IBSA-GENEVRIER, grants and personal fees from MYLAN, grants and personal fees from RADIUS HEALTH, personal fees from PIERRE FABRE, grants and personal fees from CNIEL, personal fees from DAIRY RESEARCH COUNCIL (DRC); prof. Punzi received consultancy and speaker fees from BMS, Fidia, Grunenthal, Menarini, MSD, Pfizer; Professor Cyrus Cooper has received lecture fees and honoraria from Amgen, Danone, Eli Lilly, GSK, Medtronic, Merck, Nestlé, Novartis, Pfizer, Roche, Servier, Shire, Takeda and UCB. These authors reported that these grants and personal fees are outside the submitted work. The other authors have nothing to disclose.

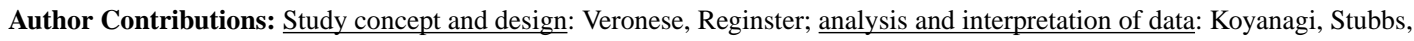
Cooper; preparation of manuscript: Veronese, Guglielmi, Maggi; critical revision: Al-Daghri, Rizzoli, Reginster, Maggi. All the authors approved the final version.
} 
University, Cambridge, UK ${ }^{17}$ Department of Public Health, Epidemiology and Health Economics, University of Liege, CHU Sart Tilman B23, 4000, Liège, Belgium

\section{Abstract}

Objectives-Mediterranean diet has several beneficial effects on health, but data regarding the association between Mediterranean diet and knee osteoarthritis (OA) are limited mainly to crosssectional studies. We investigated whether higher Mediterranean diet adherence is prospectively associated with lower risk of radiographic OA (ROA), radiographic symptomatic knee OA (SxOA) and pain worsening in North American people at high risk or having knee OA.

Methods-Adherence to the Mediterranean diet was evaluated using a validated Mediterranean diet score (aMED), categorized in five categories. Knee OA outcomes included incident (1) ROA, (2) SxOA, as the new onset of a combination of a painful knee and ROA, (3) knee pain worsening, i.e. a Western Ontario and McMaster Universities Osteoarthritis Index difference between baseline and each annual exam of $\geq 14 \%$.

Results—4,330 subjects (mean age: 61.1 years; $58.0 \%$ females) were included. Based on a multivariable Poisson regression analysis, during a mean follow-up period of 4 years, participants who were more highly adherent to a Mediterranean diet (Q5) reported lower risk of pain worsening (relative risk, $\mathrm{RR}=0.96$; 95\% CI: 0.91-0.999) compared to those in Q1. In 2,994 people free from SxOA at baseline, higher adherence to a Mediterranean diet was associated with a lower risk for SxOA during follow-up by 9\% (Q5 vs. Q1; RR=0.91; 95\%CI: 0.82-0.998). No significant associations emerged between aMED and incident ROA.

Conclusion-Higher adherence to Mediterranean diet is associated with a lower risk of pain worsening and symptomatic forms of knee OA.

\section{Keywords}

osteoarthritis; Mediterranean diet; aged; Osteoarthritis Initiative; pain; knee osteoarthritis

\section{Introduction}

With the term "Mediterranean diet", we commonly refer to the dietary habits typical of people living in the Mediterranean basin. [1] A growing body of literature suggests that the Mediterranean diet has beneficial effects on several health outcomes, including cardiovascular disease [2], metabolic syndrome [3], diabetes [4], but also musculoskeletal conditions such as osteoporosis/fracture [5], frailty [6] and sarcopenia. [7]

However, data regarding the possible association between Mediterranean diet and knee osteoarthritis (OA) are very limited. Knee OA is one of the most important contributors to global disability worldwide [8] and its prevalence is overall increasing, particularly in older people, among whom $10 \%$ of men and $20 \%$ of women over the age of 60 years suffer from this condition. $[9,10]$ Despite this, to the best of our knowledge, only a few cross-sectional studies have studied the association between higher adherence to Mediterranean diet and knee OA, showing overall that people following more strictly Mediterranean diets have a 
significantly lower likelihood of knee OA and a better knee structure. [11, 12] The Mediterranean diet is rich in vitamins and minerals, particularly those derived from vegetables and fruits, whole-meal cereals, nuts, virgin olive oil and fish, which made the risk of deficient micronutrient intakes quite infrequent. This explains why Mediterranean diet is particularly rich in antioxidant vitamins (vitamins $\mathrm{E}$ and $\mathrm{C}$ ) and carotenes were also high. [13] In this regard, other research indirectly supported a role of Mediterranean diet on knee OA. For example, in a large observational study it was found that participants introducing higher vitamin $\mathrm{C}$ and $\mathrm{E}$ and $\beta$-carotene in their diet were less likely to have knee OA. [14] However, cross-sectional studies can suffer "reverse causality" and, consequently, longitudinal cohort studies are needed to confirm the potential association between Mediterranean diet and knee OA outcomes.

Given this background, we aimed to investigate whether higher adherence to Mediterranean diet is associated with lower risk of radiographic OA (ROA), radiographic symptomatic knee $\mathrm{OA}(\mathrm{SxOA})$ and pain worsening due to knee OA, in a large cohort of North American people followed-up for 4 years.

\section{Materials and Methods}

\section{Data source and subjects}

Data were obtained from the Osteoarthritis Initiative (OAI) database. Participants were recruited across four clinical sites in the United States of America (Baltimore, MD; Pittsburgh, PA; Pawtucket, RI; and Columbus, OH) between February 2004 and May 2006. In the OAI, individuals were included if they: (1) had knee OA with knee pain for a 30-day period in the past 12 months or (2) were at high risk of developing knee OA (e.g. obese/ overweight, familiarity for knee OA).[15] The present study used data collected during the baseline/screening evaluation and in subsequent evaluations until four years of follow-up. All participants provided written informed consent. The OAI study was given full ethics approval by the institutional review board of the OAI Coordinating Center, at the University of California in San Francisco.

\section{Exposure variable: Adherence to Mediterranean diet}

Participants' diet patterns were collected using the Block Brief 2000 food frequency (FFQ) questionnaire during the baseline appointment.[16] The validated tool, containing a food list of 70 parameters, was designed to assess individuals' food and beverage consumption over the past year. Frequency of food consumption of the items included was reported at nine levels of intake from "never" to "every day". There were also seven dietary behavior questions on food preparation methods and fat intake, one question on fiber intake, and 13 questions on vitamin and mineral intakes.

Adherence to a Mediterranean diet was evaluated by applying the Mediterranean diet score (aMED) proposed by Panagiotakos et al.[17] to the FFQ. The aMED takes into consideration foods commonly consumed by Mediterranean populations. Respondents were asked to rate their consumption of each single food item using a score ranging from 0 (less adherence) to 5 (better adherence); the total possible score ranges from 0 to 55, with higher 
values indicating higher adherence to a Mediterranean diet. Cereals (e.g. bread, pasta, rice), potatoes, fruits, vegetables, legumes (e.g. peas, beans), fish were categorized on the basis of servings/month and specifically as: $0=$ never; $1=1$ to 4 servings/month; $2=5$ to $8 ; 3=9$ to 12 ; $4=13$ to $18 ; 5=$ more than 18 servings/month. As no distinction was made with reference to whole vs. refined cereals, all types of grains were considered under the same heading. Consumption of red meat, poultry, and full fat dairy products (e.g. milk cheese, yogurt) were categorized as: $0=$ more than 18 servings/month; $1=13$ to 17 servings/month; $2=9$ to $12 ; 3=5$ to $8 ; 4=1$ to $4 ; 5=$ never). Consumption of olive oil was categorized as the times it was used in a week's time and specifically as: $0=$ never; 1 =rare; $2 \leq 1$ weekly; $3=2$ times/weekly; $4=3$ to 6; 5=daily. Alcoholic beverages were categorized as: $0 \geq 700 \mathrm{ml} /$ day or $0 ; 1=600$ to 699 $\mathrm{ml} /$ day; 2=500 to $599 \mathrm{ml} /$ day; 3=400 to $499 \mathrm{ml} /$ day; 4=300 to $399 \mathrm{ml} /$ day; $5=<300 \mathrm{ml} /$ day.

The aMed score was divided into 5 quintiles: aMED score $\underline{2} 4=\mathrm{Q} 1,25-27=\mathrm{Q} 2,28-30=$ $\mathrm{Q} 3,31-32=\mathrm{Q} 4$ and $>32=\mathrm{Q} 5$. We also modelled aMED as continuous using an increase in one $\mathrm{SD}$ (i.e. 5 points).

\section{Assessment of knee OA outcomes}

At baseline and during follow-up examinations, people had full knee assessments which included both a clinical and radiographic examination. Individuals with knee replacement at baseline were removed from analyses. A fixed flexion posterior-anterior radiograph, which was read centrally for Kellgren and Lawrence (KL) grade, was made for all the participants. In addition, participants were asked regarding knee pain, the following question: 'During the past 30 days, have you had pain, aching, or stiffness in your right/left knee on most days?'. Knee pain was also assessed using the WOMAC (Western Ontario and McMaster Universities Osteoarthritis Index) pain subscale. This scale ranges from 0 (absence of pain) to 20 (highest pain) points.

In the present study the following knee outcomes were considered: (1) incident ROA (radiographical osteoarthritis), if a knee developed a KL grade $\geq 2$ at follow-up and was not present at baseline, (2) SxOA (symptomatic osteoarthritis), defined as new onset of a combination of painful knee plus (determined using the answer yes to the following question: "During the past 30 days, have you had pain, aching, or stiffness in your right/left knee on most days?") together the presence of ROA. Both these criteria should be present at the same site and (3) knee pain worsening, i.e. WOMAC pain score difference of a knee between baseline and each annual exam $\geq 14 \%$ or the onset of any pain during follow-up in people having zero at baseline in agreement with previous research regarding this topic.[18$20]$

The assessment of these knee OA outcomes was made, other than at baseline, at V01 (12 months), V03 (24 months), V05 (36 months), and V06 (48 months).

\section{Covariates}

Ten covariates at baseline (other than age and sex) were identified as potential confounding factors based on previous literature.[21] These included: race (whites vs. others); educational attainment (college or higher vs. others); body mass index (BMI); yearly income (< vs $\geq \$ 50,000$ or missing data); depressive symptoms measured using the Center for 
Epidemiologic Studies Depression Scale (CES-D)[22]; smoking habits (current and previous vs. never); physical activity level evaluated using the Physical Activity Scale for the Elderly (PASE) [23]; Charlson Comorbidity Index score [24]; daily energy intake; number of medications at baseline.

\section{Statistical analyses}

Continuous variables were normally distributed according to the Kolmogorov-Smirnov test. Data were presented as means and standard deviation values (SD) for quantitative measures, and percentages for all categorical variables by aMED scores. $\mathrm{P}$ values for trends were calculated using the Jonckheere-Terpstra test for continuous variables and the MantelHaenszel Chi-square test for categorical variables.

To assess associations between aMED and the outcomes ROA, SxOA, and pain worsening, a multivariable Poisson regression analysis with robust variance estimators was applied, since a survival analysis was not possible due to lack of information on the precise date of event. We used two models, one not adjusted for any covariate, and one fully-adjusted using the aforementioned covariates. Multi-collinearity among covariates was assessed through variance inflation factor [25], taking a cut-off of 2 as the criterion for exclusion. However, no covariates were excluded using this criterion. Relative risks (RRs) and 95\% confidence intervals (CI) were calculated to estimate the strength of the associations between aMED and incident knee OA outcomes.

A $p<0.05$ was deemed statistically significant. All analyses were performed using SPSS ${ }^{\circledR}$ software version 17.0 for Windows (SPSS Inc., Chicago, Illinois) and STATA ${ }^{\circledR}$ software version 14.1 (Stata Corp LP, College station, Texas).

\section{Results}

\section{Sample selection}

The OAI dataset initially included a total of 4,796 individuals. We excluded 63 participants with a total knee replacement at baseline (since they cannot develop any of the outcomes of interest), 118 not having sufficient information for calculating aMED or having implausible calorie intake ( $<500$ or $>4000 \mathrm{Kcal})$ and a further 285 with no data regarding outcomes of interest during follow-up. Thus, the final sample consisted of 4,330 people $(=90.2 \%$ of the entire population initially involved in the OAI).

\section{Descriptive characteristics}

The baseline cohort consisted of 2,510 females (58.0\%) and 1,820 males (42.0\%), with a mean age of 61.1 years $( \pm 9.2$ years; range: $45-79$ years). The mean aMED was 28 points ( \pm 5 points; range: $0-44)$. At baseline, 2,491 had knee ROA (=57.5\% of baseline population) and, of them, 1,078 had knee SxOA (=24.9\%).

Table 1 shows the baseline characteristics by aMED score category. People more strictly following Mediterranean diet (i.e. included in Q5) were significantly older, more frequently females and whites, more frequently physical active and educated than those having lower 
scores (=Q1). Moreover, those in Q5 were less obese and depressed, reported lower presence of medical conditions and used less medications than those included in the first category.

\section{Mediterranean diet adherence and incident knee osteoarthritis outcomes}

As shown in Table 2, during a mean follow-up period of 4 years, after adjusting for several potential confounders at baseline, participants in Q5 (i.e. those having a higher adherence to Mediterranean diet) reported a significantly lower risk for pain worsening $(\mathrm{RR}=0.96$; 95\%CI: 0.91-0.999; $\mathrm{p}=0.047$ ) compared to those in Q1. Each increase in one SD (i.e. 5 points) was associated with a significantly lower risk of pain worsening by $2 \%$ ( $R R=0.98$; 95\%CI: 0.97-0.998; $\mathrm{p}=0.04$ ) (Table 2).

In 2,994 subjects free from SxOA at the baseline, higher adherence to Mediterranean diet was associated with a significantly lower risk for SxOA during follow-up by $9 \%$ (RR=0.91; 95\%CI: 0.82-0.998; $\mathrm{p}=0.048$ ) and each one SD increase in aMED lowered the risk for this outcome by $4 \%$.

On the contrary, no significant associations emerged between aMED and incident ROA, as shown in Table 2.

\section{Discussion}

In this large longitudinal study over a 4-year follow-up period, our results suggest that higher adherence to Mediterranean diet is associated with a significantly lower risk of pain worsening and symptomatic knee OA, whilst no significant effect was observed on the incidence of radiographic knee OA.

Although knee OA is a common condition and diet seems to play a pivotal role in the prevention and in supporting the treatment of this condition [26], the data regarding the potential association between higher adherence to Mediterranean diet and knee OA are, unfortunately, limited. In the Osteoarthritis Initiative, we have already reported that higher aMED scores were not only associated with a significant lower prevalence of knee OA (also after accounting for potential confounders) [12], but also with a better architecture of the knee, investigated through a magnetic resonance investigation.[11] However these data were cross-sectional limiting the interpretation of these findings. With the current research, we have further reinforced the concept that Mediterranean diet could be useful for the prevention of pain in knee OA and for the symptomatic forms through a longitudinal design.

We can hypothesize several mechanisms that may explain the association between adherence to Mediterranean diet and reduction in pain/symptomatic knee OA. First, a stricter adherence to a Mediterranean diet has been shown to be associated with an important decrease in inflammatory parameters [27] and it is widely known that inflammation plays a pivotal role in the destruction of knee cartilage. [28] This effect (i.e. anti-inflammatory) seems to be the most important factor in the link between Mediterranean diet and knee OA, as also confirmed by a small randomized controlled trial showing that people following a Mediterranean diet regimen had a significant decrease in IL1a, a potent pro-inflammatory cytokine.[29] In this sense, the anti-inflammatory properties typical of a Mediterranean diet 
may consequently reduce pain and symptomatic forms of knee OA. Similarly, following a Mediterranean diet seems to lower oxidative stress markers [30], which may influence the onset of symptomatic OA by enhancing collagen type II and aggrecan expression levels and inhibiting apoptosis-related protein expression. [30] Third, the Mediterranean diet is particularly rich in some nutrients that may have a protective effect on knee OA outcomes, such as fibers and vitamins. For example, a previous study reported that dietary fibers, particularly present in Mediterranean diet, are able to prevent/improve several knee OA outcomes. [19] Finally, Mediterranean diet seems to have a role in improving the extracellular matrix (ECM) [30], consequently promoting the effective repair of a structure which is frequently reduced in people developing knee OA. In animal models, it was reported that the use of olive oil, a pivotal component of Mediterranean diet, significantly improved articular cartilage structure, indicating a potential role of diets rich in this component for knee OA outcomes. [31]

The long duration of follow-up, the multiple knee OA outcomes included, and the large sample size are clear strengths of the present study. However, findings from the present study should be interpreted in light of its limitations. First, participants recruited into the OAI were either at high risk of developing or already had knee OA. Thus, further research in the general population is needed to confirm our findings. Second, our findings are observational and, although we tried to overcome this limitation using analyses adjusted for potential confounders, we cannot exclude potential bias. In this sense, of particular importance is that dietary questionnaires can suffer recall bias.[32] Finally, the record of medications and comorbidities is self-reported and, again, we cannot exclude a potential bias for these parameters.

In conclusion, our data suggest that, higher adherence to Mediterranean diet is associated with a lower risk of pain worsening and symptomatic forms of knee OA, whilst no significant association emerged when taking radiographic knee OA as outcome. Although our research provides further evidence on the important role of Mediterranean diet on knee OA outcomes, future RCTs are needed to confirm or refute our findings.

\section{Acknowledgements}

Founding source: The OAI is a public-private partnership comprised of five contracts (N01-AR-2-2258; N01AR-2-2259; N01-AR-2-2260; N01-AR-2-2261; N01-AR-2-2262) funded by the National Institutes of Health, a branch of the Department of Health and Human Services, and conducted by the OAI Study Investigators. Private funding partners include Merck Research Laboratories; Novartis Pharmaceuticals Corporation, GlaxoSmithKline; and Pfizer, Inc. Private sector funding for the OAI is managed by the Foundation for the National Institutes of Health. This manuscript was prepared using an OAI public use data set and does not necessarily reflect the opinions or views of the $\mathrm{OAI}$ investigators, the $\mathrm{NIH}$, or the private funding partners.

Sponsor's Role: none.

\section{References}

1. Willett WC, Sacks F, Trichopoulou A, Drescher G, Ferro-Luzzi A, Helsing E, et al. Mediterranean diet pyramid: A cultural model for healthy eating. 1995

2. Dinu M, Pagliai G, Casini A, Sofi F. Mediterranean diet and multiple health outcomes: an umbrella review of meta-analyses of observational studies and randomised trials. European journal of clinical nutrition. 2018 Jan; 72(1):30-43. [PubMed: 28488692] 
3. Babio N, Bulló M, Salas-Salvadó J. Mediterranean diet and metabolic syndrome: the evidence. Public health nutrition. 2009; 12(9A):1607-1617. [PubMed: 19689829]

4. Salas-Salvadó, JbMBN. Reduction in the Incidence of Type 2 Diabetes With the Mediterranean Diet. Diabetes care. 2011; 34(1):14-19. [PubMed: 20929998]

5. Puel C, Coxam V, Davicco M-J. Mediterranean diet and osteoporosis prevention. Medecine sciences: M/S. 2006; 23(8-9):756-760.

6. Veronese N, Stubbs B, Noale M, Solmi M, Rizzoli R, Vaona A, et al. Adherence to a Mediterranean diet is associated with lower incidence of frailty: A longitudinal cohort study. Clinical nutrition (Edinburgh, Scotland). 2017 Sep 4.

7. Kelaiditi E, Jennings A, Steves CJ, Skinner J, Cassidy A, MacGregor AJ, et al. Measurements of skeletal muscle mass and power are positively related to a Mediterranean dietary pattern in women. Osteoporosis international : a journal established as result of cooperation between the European Foundation for Osteoporosis and the National Osteoporosis Foundation of the USA. 2016 Nov; 27(11):3251-3260.

8. van Dijk GM, Veenhof C, Lankhorst GJ, Dekker J. Limitations in activities in patients with osteoarthritis of the hip or knee: the relationship with body functions, comorbidity and cognitive functioning. Disability and rehabilitation. 2009; 31(20):1685-1691. [PubMed: 19479564]

9. O'Connor MI. Sex differences in osteoarthritis of the hip and knee. The Journal of the American Academy of Orthopaedic Surgeons. 2007; 15(Suppl 1):S22-25. [PubMed: 17766785]

10. Duncan R, Francis RM, Collerton J, Davies K, Jagger C, Kingston A, et al. Prevalence of arthritis and joint pain in the oldest old: findings from the Newcastle 85+ study. Age and ageing. 2011; 40(6):752-755. [PubMed: 21937515]

11. Veronese N, La Tegola L, Crepaldi G, Maggi S, Rogoli D, Guglielmi G. The association between the Mediterranean diet and magnetic resonance parameters for knee osteoarthritis: data from the Osteoarthritis Initiative. Clinical rheumatology. 2018 Apr 3.

12. Veronese N, Stubbs B, Noale M, Solmi M, Luchini C, Smith TO, et al. Adherence to a Mediterranean diet is associated with lower prevalence of osteoarthritis: Data from the osteoarthritis initiative. Clinical nutrition (Edinburgh, Scotland). 2017 Dec; 36(6):1609-1614.

13. Castro-Quezada I, Román-Viñas B, Serra-Majem L. The Mediterranean diet and nutritional adequacy: a review. Nutrients. 2014; 6(1):231-248. [PubMed: 24394536]

14. McAlindon T, Zhang Y, Hannan M, Naimark A, Weissman B, Castelli W, et al. Are risk factors for patellofemoral and tibiofemoral knee osteoarthritis different? Journal of Rheumatology. 1996; 23(2):332-337. [PubMed: 8882042]

15. Eby GA, Eby KL. Rapid recovery from major depression using magnesium treatment. Medical Hypotheses. 2006; 67(2):362-370. [PubMed: 16542786]

16. Willett WC, Sampson L, Stampfer MJ, Rosner B, Bain C, Witschi J, et al. Reproducibility and validity of a semiquantitative food frequency questionnaire. American journal of epidemiology. 1985; 122(1):51-65. [PubMed: 4014201]

17. Panagiotakos DB, Pitsavos C, Stefanadis C. Dietary patterns: a Mediterranean diet score and its relation to clinical and biological markers of cardiovascular disease risk. Nutrition, metabolism, and cardiovascular diseases : NMCD. 2006; 16(8):559-568.

18. Angst F, Aeschlimann A, Michel BA, Stucki G. Minimal clinically important rehabilitation effects in patients with osteoarthritis of the lower extremities. The Journal of rheumatology. 2002 Jan; 29(1):131-138. [PubMed: 11824949]

19. Dai Z, Niu J, Zhang Y, Jacques P, Felson DT. Dietary intake of fibre and risk of knee osteoarthritis in two US prospective cohorts. Annals of the rheumatic diseases. 2017 Aug; 76(8):1411-1419. [PubMed: 28536116]

20. Veronese N, Koyanagi A, Stubbs B, Cooper C, Guglielmi G, Rizzoli R, et al. Statin use and knee osteoarthritis outcomes: A longitudinal cohort study. Arthritis care \& research. 2018 Aug 24.

21. Allen KD, Golightly YM. Epidemiology of osteoarthritis: state of the evidence. Current opinion in rheumatology. 2015; 27(3):276-283. [PubMed: 25775186]

22. Lewinsohn PM, Seeley JR, Roberts RE, Allen NB. Center for Epidemiologic Studies Depression Scale (CES-D) as a screening instrument for depression among community-residing older adults. Psychology and aging. 1997; 12(2):277-287. [PubMed: 9189988] 
23. Washburn RA, McAuley E, Katula J, Mihalko SL, Boileau RA. The physical activity scale for the elderly (PASE): evidence for validity. Journal of clinical epidemiology. 1999; 52(7):643-651. [PubMed: 10391658]

24. Katz JN, Chang LC, Sangha O, Fossel AH, Bates DW. Can comorbidity be measured by questionnaire rather than medical record review? Medical care. 1996; 34(1):73-84. [PubMed: 8551813]

25. Miles, J. Tolerance and variance inflation factorWiley StatsRef: Statistics Reference Online. 2009.

26. Litwic A, Edwards MH, Dennison EM, Cooper C. Epidemiology and burden of osteoarthritis. British Medical Bulletin. 2013; 105(1):185-199. [PubMed: 23337796]

27. Chrysohoou C, Panagiotakos DB, Pitsavos C, Das UN, Stefanadis C. Adherence to the Mediterranean diet attenuates inflammation and coagulation process in healthy adults: The ATTICA Study. Journal of the American College of Cardiology. 2004; 44(1):152-158. [PubMed: 15234425]

28. Rainbow R, Ren W, Zeng L. Inflammation and Joint Tissue Interactions in OA: Implications for Potential Therapeutic Approaches. Arthritis. 2012; 2012

29. Dyer J, Davison G, Marcora SM, Mauger AR. Effect of a Mediterranean Type Diet on Inflammatory and Cartilage Degradation Biomarkers in Patients with Osteoarthritis. The journal of nutrition, health \& aging. 2017; 21(5):562-566.

30. Scoditti E, Calabriso N, Massaro M, Pellegrino M, Storelli C, Martines G, et al. Mediterranean diet polyphenols reduce inflammatory angiogenesis through MMP-9 and COX-2 inhibition in human vascular endothelial cells: A potentially protective mechanism in atherosclerotic vascular disease and cancer. 2012:81-89.

31. Musumeci G, Trovato FM, Pichler K, Weinberg AM, Loreto C, Castrogiovanni P. Extra-virgin olive oil diet and mild physical activity prevent cartilage degeneration in an osteoarthritis model: An in vivo and in vitro study on lubricin expression. Journal of Nutritional Biochemistry. 2013; 24(12):2064-2075. [PubMed: 24369033]

32. Kipnis V, Midthune D, Freedman L, Bingham S, Day NE, Riboli E, et al. Bias in dietary-report instruments and its implications for nutritional epidemiology. Public health nutrition. 2002; 5(6a): 915-923. [PubMed: 12633516] 


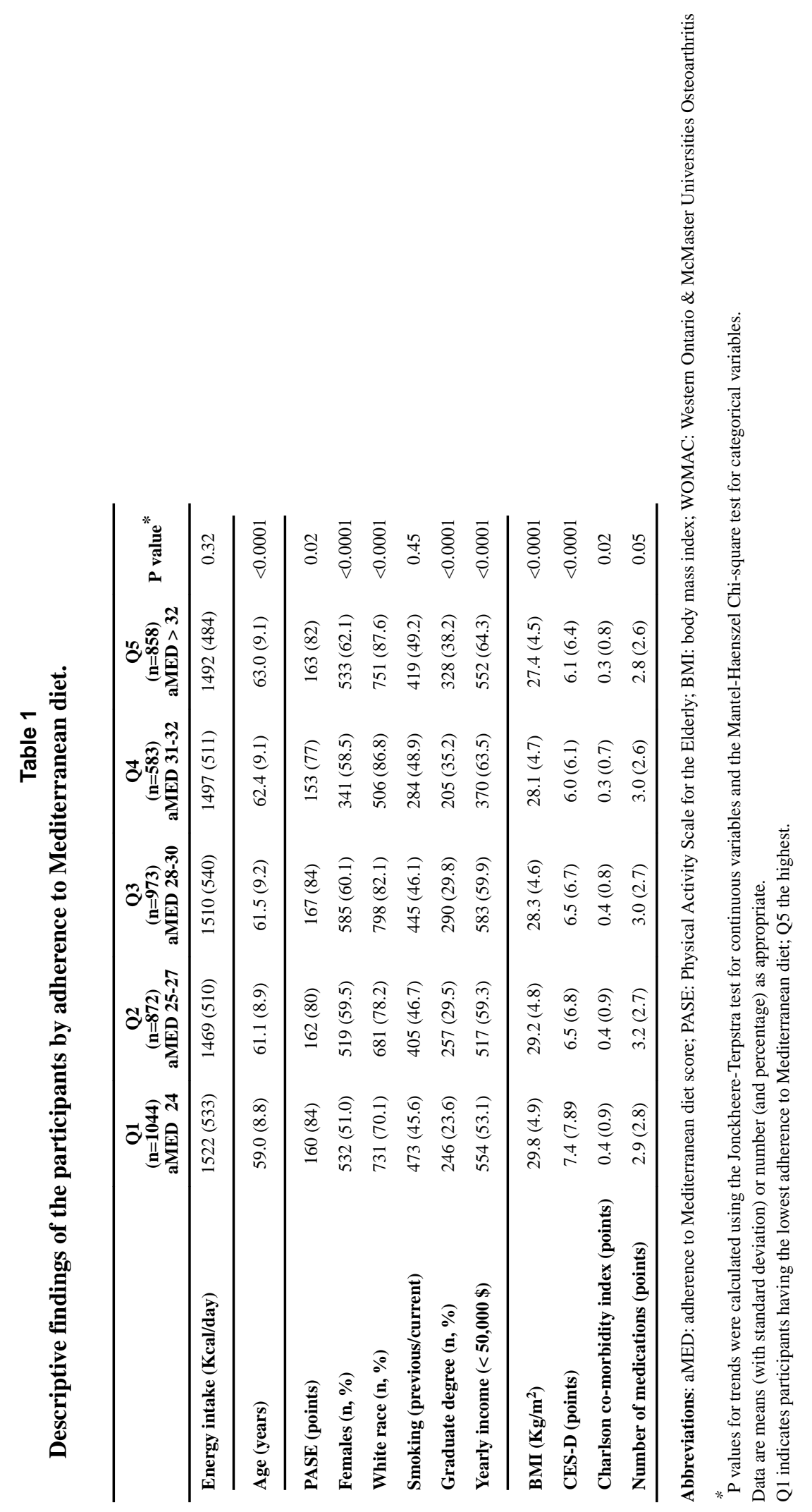




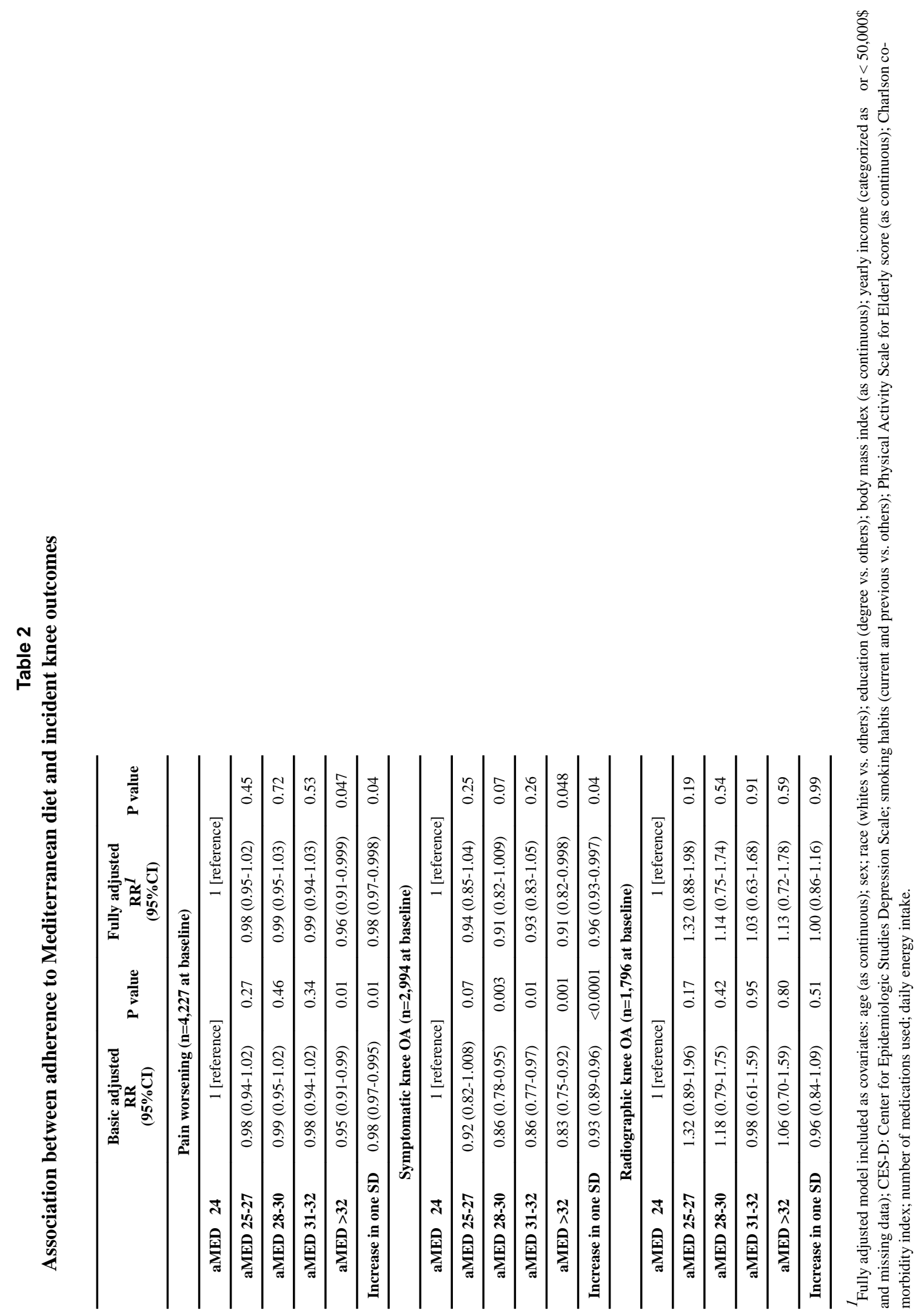

